\title{
Zwischen Pest und Cholera?
}

$\mathrm{G}$ erade wurde das zehnjährige Jubiläum der Europäischen Währungsunion $\mathcal{Y}_{(\mathrm{EWU})}$ gefeiert, schon wird in den Medien über ihren Zerfall spekuliert. Ist das Panikmache oder nur Folge der Finanzkrise, in der alles viel kritischer auf den Prüfstand gestellt wird als zuvor? Wie kommt es, dass man von offizieller Seite kaum klare Stellungnahmen hört? Haben die Hüter der Währung, die Hüterin der Verträge (die Europäische Kommission) und der Europäische Rat (die Finanzminister) die Warnungen nicht gehört oder nehmen sie sie nicht ernst? Im letzten Fall wäre es angemessen, klare Dementis zu verbreiten, die helfen, die Gerüchte zu zerstreuen oder unzweifelhaft zu widerlegen. Oder haben sich gar alle diese Institutionen über die wahre Lage getäuscht? Offenbar genügt Preisstabilität, wie sie im Durchschnitt aller Mitgliedsländer in den zurückliegenden zehn Jahren erreicht wurde, nicht aus, um die Währungsunion zu einem Erfolg zu machen.

Anders als in den Jahren vor der EWU haben sich die Inflationsraten der Mitgliedsländer nach deren Beginn und in der Phase der gleichartigen Geldpolitik nicht weiter angeglichen. Das schon war der erste Tiefschlag, widerlegte es doch die Geldmengentheoretiker, die fest daran geglaubt hatten, dass bei einheitlicher Geldpolitik auch die Inflationsentwicklung einheitlich sein müsse. Allmählich erkannte man zwar die überragende Bedeutung, die die Entwicklung der nationalen gesamtwirtschaftlichen Lohnstückkosten für die nationalen Inflationsraten hat. Die naheliegenden Schlussfolgerungen aber wollte man nicht ziehen. Nominallohn- und Produktivitätswachstum, die die Entwicklung der Lohnstückkosten bestimmen, unterliegen nämlich - anders als sich das Geldmengentheoretiker für eine Steuerung der Inflationsrate wünschen - nicht dem direkten Einfluss der Zentralbank, sondern werden zum einen von den Tarifparteien ausgehandelt und zum anderen von den Sachinvestoren auf die Beine gestellt.

Die zweite Kröte zu schlucken, war noch schwieriger: Angesichts ihres festen Glaubens an die neoklassische Beschäftigungstheorie wurde Deutschland von der EZB und der Deutschen Bundesbank stets für seine moderate Lohnpolitik gelobt und den anderen EWU-Mitgliedsländern als Vorbild empfohlen. Da die Zentralbanker überzeugt waren und sind, dass es ohne Lohnzurückhaltung keinen Abbau der Arbeitslosigkeit gibt, konnten und wollten sie dem deutschen Experiment in Sachen Neoklassik die Unterstützung nicht verweigern. Zudem wäre ohne deutsches „Gürtel enger Schnallen“ die Verfehlung des 2\%-Inflationsziels der EZB an der Tagesordnung gewesen. Die größte europäische Volkswirtschaft sorgte mit ihren seit Jahren stagnierenden Reallöhnen und Lohnstückkosten für Preisstabilität in Euro-Land - aber eben nur im Durchschnitt des Währungsraums.

Die Kehrseite der divergierenden Preisentwicklungen in der EWU sind der enorme deutsche Handelsüberschuss mit den EWU-Partnern und die Marktanteilsgewinne Deutschlands auf Drittmärkten. Das unterdurchschnittliche Wachstum der deutschen Lohnstückkosten hat uns riesige Wettbewerbsvorteile verschafft und die EWU-Partner, die sich dem nicht durch eine Abwertung ihrer Währung entziehen konnten, in die Auslandsverschuldung getrieben. Die hat inzwischen ein Ausmaß angenommen, das ein Währungsrisiko schafft, weil die Kapitalmärkte auf ein Auseinanderbrechen der EWU spekulieren. Ein Indikator hierfür sind die zunehmenden Zinsdifferenzen auf Staatsanleihen innerhalb der EWU.

Damit ist das Argument hinfällig, auf dem bisher die Abwehrstrategie von EZB, der Bundesbank und des deutschen Sachverständigenrates beruhte. Weil nämlich Länder mit relativ hoher Inflationsrate im Euroraum relativ geringe Realzinsen

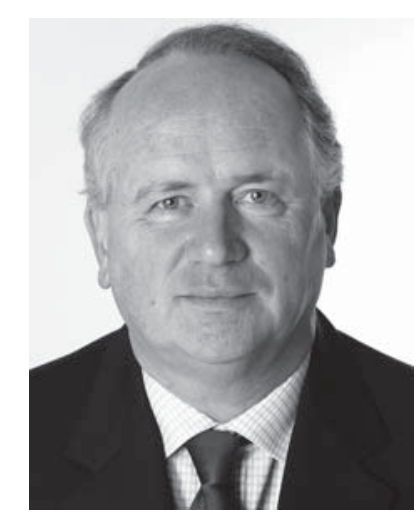

Heiner Flassbeck 


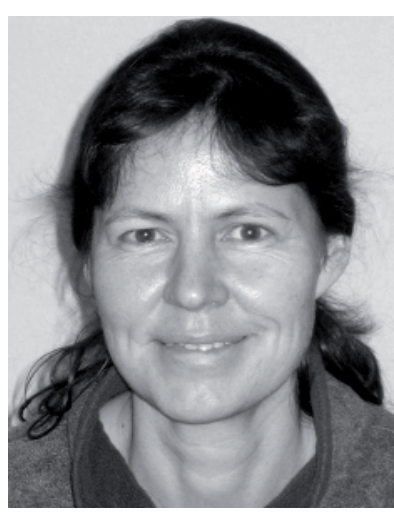

Friederike Spiecker aufwiesen, so die einhellige Meinung dieser Experten, könnten dadurch die Verluste bei der Wettbewerbsfähigkeit ausgeglichen werden. Das war zwar schon immer falsch, weil „eine gegebene Inflationsdifferenz bei identischen Nominalzinsen eine lediglich konstante Realzinsdifferenz (impliziert), während die preisliche Wettbewerbsfähigkeit in dem Land mit der niedrigen Teuerungsrate kontinuierlich zunimmt“ und „So gesehen wird der Effekt verbesserter Wettbewerbsfähigkeit bei anhaltenden Inflationsunterschieden mit der Zeit an Kraft gewinnen“, wie im Monatsbericht der Bundesbank in der Dezemberausgabe 2008 zu lesen ist.

Diese wunderbare Erkenntnis, die nach einigen Jahren der Verwirrung nun auch die Deutsche Bundesbank gewonnen hat, hindert die Hüterin der europäischen Währung in Deutschland aber nicht daran, den deutschen Weg weiter zu verteidigen. Trotz der offensichtlichen Gefahr, die von ihr ausgeht, wird die deutsche Lohnpolitik bis heute von offizieller Seite nicht als Ursache der Spannungen in der EWU genannt. Im Gegenteil, wenige Seiten später empfiehlt die Deutsche Bundesbank die deutsche Beggar-thy-neighbour-Strategie weiterhin unbekümmert: „... unter den Spielregeln einer Währungsunion (gibt es) keine effektiven, nachhaltigen und zugleich marktkonformen wirtschaftspolitischen Alternativen zu dem hierzulande beschrittenen Weg. Insofern ist die deutsche Erfahrung mit Blick auf andere EWU-Länder, die mit dem Problem einer abnehmenden preislichen Wettbewerbsfähigkeit konfrontiert sind, durchaus als exemplarisch zu bezeichnen.“

Die hoch verschuldeten EWU-Mitgliedsländer werden also zu einer lohninduzierten Deflationspolitik nach deutschem Vorbild aufgerufen, um ihre Probleme zu lösen. In den Augen der Zentralbanker ist das offenbar völlig ungefährlich, da sie die „hinreichende Lohnflexibilität nach unten“ nebenbei als „zentrale(n) Faktor ... für die Stärkung der heimischen Wachstumskräfte“ ansehen. Von der damit eindeutig verbundenen Gefahr einer massiven Abweichung vom Ziel der Preisstabilität - nämlich nach unten - ist nicht die Rede.

Die EWU muss sich also, folgt sie der Ansicht der Deutschen Bundesbank, zwischen Auseinanderbrechen und Deflationsspirale entscheiden. Wann werden die Hüter der Währung zu einer vorurteilsfreien Analyse fähig sein, aus der Lohnempfehlungen folgen, die mit dem Ziel einer Inflationsrate von etwa $2 \%$ in Einklang stehen und der EWU mehr als die Wahl zwischen Pest und Cholera bieten? Wann wird die deutsche Wirtschaftspolitik verstehen, dass eine enge internationale wirtschaftliche Verflechtung zum gegenseitigen Nutzen nur möglich ist, wenn sie mit tendenziell ausgeglichenen Leistungsbilanzen einhergeht? Wann wird man erkennen und offen sagen, dass eine Währungsunion nur funktionieren kann, wenn jedes Mitgliedsland dafür sorgt, dass seine Nominallöhne in Höhe des nationalen Produktivitätsfortschritts plus der Zielinflationsrate der Zentralbank wachsen - nicht mehr, aber auch nicht weniger?

Will man die Europäische Währungsunion noch vor dem Untergang retten, reicht die Besinnung auf diese einfachen Regeln aber nicht. Um die Wettbewerbsverhältnisse wieder auszugleichen, also zumindest die absoluten Preise anzugleichen (von einer Wiederherstellung der alten Marktanteilsstrukturen gar nicht zu reden), müssen über viele Jahre die deutschen Löhne deutlich über dem Normalpfad wachsen und die der anderen müssen darunter bleiben. Der politische Kraftakt, der notwendig wäre, um so etwas durchzusetzen und dem staunenden Publikum zu erklären, lässt wohl alle Beteiligten derart schaudern, dass sie es gar nicht erst versuchen.

Heiner Flassbeck ist Direktor einer Division der UNCTAD in Genf; Friederike Spiecker ist Diplom-Volkswirtin und freie Wirtschaftspublizistin www.flassbeck.de 